\title{
User Privacy Protection in Personalised Web Search
}

\author{
Siva $B^{1}$, Merlin Shoerio ${ }^{2}$ \\ ${ }^{1}$ M.Tech Student, Marian Engineering College, Trivandrum, Kerala, India \\ ${ }^{2}$ Assistant Professor, Department of CSE, Marian Engineering College, Trivandrum, Kerala, India
}

\begin{abstract}
Today's world is depending more on the internet for knowing new things or for clearing the doubts related to their field of work etc. But when we enter a topic in the search engine it is not necessary that the pages related to our interested area would come. It may show the pages in various fields that possess that word. So sometimes it may be irritating for the users when the pages which are not of their interests are displayed. So this paper deals with the idea of personalised web search where a user profile is created. It may contain the details of the user and his topics of interests. But due to security reasons most of the users do not disclose their private information. This paper provides an online profile creation where the user can customize his related topics and when a query is entered the topics related to his interests will be displayed first with his privacy preserved.
\end{abstract}

Keywords: Privacy preservation, personalised web search, ranking, online profile creation etc.

\section{Introduction}

Most of the users now use search engines for getting valuable information related to their topics. But sometimes they may get information which is not on their topic of interests. This may make the users irritated. So it will be preferable if search results may get according to some profile or something like that where the user can customize his needs. But due to the security reasons the users are not willing to give their private information. This may affect the search results adversely. Thus for providing better search results the user information must be collected and analysed and their query must be processed according to these data.

Many methods have been proposed for better personalised web search. Some were based on the click log methods [2] where the search results were based on the previous history. These techniques give better results only for the repeated queries. So these techniques are not optimal. The other techniques are profile based ones [3][4], where an offline or online profile creation is done. For offline profile creation once the profile is created this may be used for ever. Here the user cannot customize their needs. But in online profile creation the user can customize their needs. Here arises the need of security.

\section{Related Works}

As seen before, the personalised web search is categorized into two types like click log based ones and profile based ones. In click log based ones [2], the search results are based on the previous history. In profile based ones [3][4] a profile is created for the user and the query is processed according to this profile. Among these, most works support only offline profile creation [6][8]. Here the user cannot customize his needs. Thus once the profile is created all the queries will be processed according to his once created profile. Some methods do not allow the users to prioritize [9][10]their needs. Thus all the needs will be given equal preference. All these methods require privacy preservation [6][8].

\section{System Model}

This paper deals with online profile creation[5] where the user can customize the needs according to his priority. The system model assumes a large number of clients and a server. Here the server is assumed as untrusting. When a user sends a query, the hierarchical profile of the user is created [7]. There the user can customize their needs. Then the query is generalised according to this profile. This is then send to the server side [11] and the server sends results according to the generalised query.

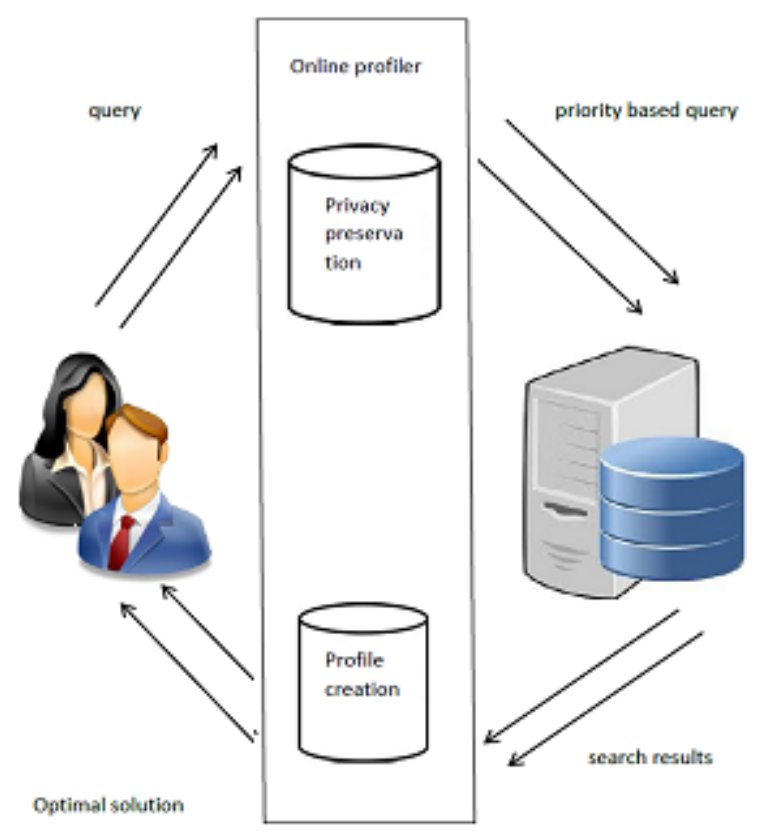

Figure 1: System Model

\section{Proposed System}

The user can customize the needs according to their priority on the online profile. Also the user can enter comments for each topic. So when a query is entered and if the user is not given any priority to that particular topic, then the comments of the user for that topic is analysed and the query will be generalised according to the priority or comments. This 


\section{International Journal of Science and Research (IJSR) \\ ISSN (Online): 2319-7064 \\ Index Copernicus Value (2013): 6.14 | Impact Factor (2015): 6.391}

generalised query will be send to the server. The server may give responses according to this generalised query. Thus an optimal solution can be obtained.

\section{Performance Analysis}

The performance of the system can be analysed based on the security it provides and the search results it provides. It will provide better search results as it give responses according to the user profile. Also it provides better security as the profile as such is not send to the server. Only a generalised query based on the profile is send to the server.

\section{Conclusion}

Users hope for better search results. Profile based ones may result in better search results. But there arise the issue of security. An optimal solution can be obtained if better security measures are given. This paper provides a better method for providing privacy protection and also better search results.

\section{References}

[1] Lidan Shou, He Bai, Ke Chan, and Gang Chen, "Supporting Privacy Protection in Personalised Web Search," IEEE Transactions on Knowledge Engineering, Vol.26, No.2, Feb 2014.

[2] Z. Dou, R. Song, and J.-R. Wen, "A Large-Scale Evaluation and Analysis of Personalized Search Strategies," Proc. Int'l Conf. World Wide Web (WWW), pp. 581-590, 2007.

[3] F. Qiu and J. Cho, "Automatic Identification of User Interest for Personalized Search," Proc. 15th Int'l Conf. World Wide Web (WWW), pp. 727-736, 2006.

[4] Y. Xu, K. Wang, G. Yang, and A.W.-C. Fu, "Online Anonymity for Personalized Web Services," Proc. 18th ACM Conf. Information and Knowledge Management (CIKM), pp. 1497-1500, 2009.

[5] G. Chen, H. Bai, L. Shou, K. Chen, and Y. Gao, "Ups: Efficient Privacy Protection in Personalized Web Search," Proc. 34th Int'l ACM SIGIR Conf. Research and Development in Information, pp. 615-624, 2011.

[6] A. Krause and E. Horvitz, "A Utility-Theoretic Approach to Privacy in Online Services," J. Artificial Intelligence Research, vol. 39, pp. 633-662, 2010.

[7] K. Ramanathan, J. Giraudi, and A. Gupta, "Creating Hierarchical User Profiles Using Wikipedia," HP Labs, 2008

[8] X. Shen, B. Tan, and C. Zhai, "Privacy Protection in Personalized Search," SIGIR Forum, vol. 41, no. 1, pp. 4-17, 2007.

[9] A. Viejo and J. Castell_a-Roca, "Using Social Networks to Distort Users' Profiles Generated by Web Search Engines," Computer Networks, vol. 54, no. 9, pp. 13431357, 2010.

[10] J. Pitkow, H. Schu“ tze, T. Cass, R. Cooley, D. Turnbull, A. Edmonds, E. Adar, and T. Breuel, "Personalized Search," Comm. ACM, vol. 45, no. 9, pp. 50-55, 2002.
[11]F. Qiu and J. Cho, "Automatic Identification of User Interest for Personalized Search," Proc. 15th Int'l Conf. World Wide Web (WWW), pp. 727-736, 2006.

Volume 5 Issue 6, June 2016 www.ijsr.net 